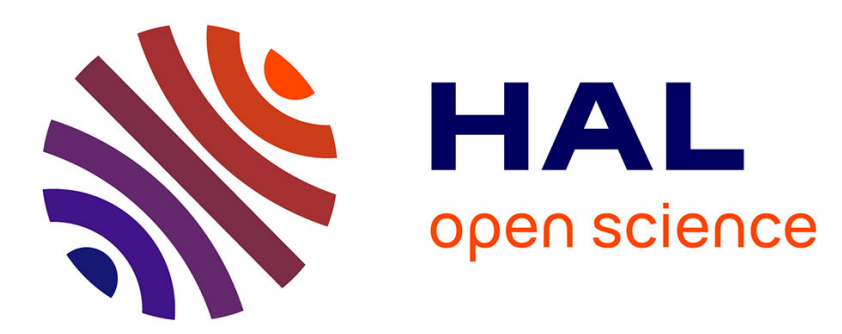

\title{
Photorefractive effect generated in sillenite crystals by picosecond pulses and comparison with the quasi continuous regime
}

\author{
Gilles Pauliat, Gérald Roosen
}

\section{- To cite this version:}

Gilles Pauliat, Gérald Roosen. Photorefractive effect generated in sillenite crystals by picosecond pulses and comparison with the quasi continuous regime. Journal of the Optical Society of America B, 1990, 7 (12), pp.2259-2267. hal-00856195

\section{HAL Id: hal-00856195 \\ https://hal-iogs.archives-ouvertes.fr/hal-00856195}

Submitted on 30 Aug 2013

HAL is a multi-disciplinary open access archive for the deposit and dissemination of scientific research documents, whether they are published or not. The documents may come from teaching and research institutions in France or abroad, or from public or private research centers.
L'archive ouverte pluridisciplinaire $\mathbf{H A L}$, est destinée au dépôt et à la diffusion de documents scientifiques de niveau recherche, publiés ou non, émanant des établissements d'enseignement et de recherche français ou étrangers, des laboratoires publics ou privés. 


\title{
Photorefractive effect generated in sillenite crystals by picosecond pulses and comparison with the quasi-continuous regime
}

\author{
G. Pauliat and G. Roosen
}

\begin{abstract}
Institut d'Optique, Unité de Recherche Associée au Centre National de la Recherche Scientifique, Bâtiment 503, B.P. 147, 91403 Orsay Cedex, France
\end{abstract}

Received May 22, 1990; accepted June 21, 1990

\begin{abstract}
Photorefractive gratings are generated in a BSO crystal by the interference of two 25-psec pulses at $532 \mathrm{~nm}$. The grating is analyzed by diffraction of a third pulse or a weak $\mathrm{cw}$ argon laser, which permits a continuous monitoring of its evolution from picoseconds to milliseconds. The grating builds up in two steps; their relative contributions vary strongly with fluence. This behavior is explained by means of a band transport model with shallow traps. Values of mobility, lifetimes, and diffusion lengths of carriers are derived and compared with those obtained in experiments conducted with low-power lasers; the values show a striking continuity from cw to picosecond regimes.
\end{abstract}

\section{INTRODUCTION}

The photorefractive effect, identified in many insulating and semiconductor crystals, has permitted the demonstration of a wide variety of operations in the fields of phase conjugation, dynamic interconnections, and optical signal processing. ${ }^{1-3}$ This nonlinear effect results from a light-induced charge redistribution in the material, which generates a space-charge electric field, which, in turn, modulates the crystal refractive index through the electrooptic effect. As a consequence, the photorefractive effect is inherently sensitive to the absorbed optical energy, permitting efficient recording of phase gratings by the interference of low-power, quasi-continuous light beams. However, to first order the time required for recording a grating of a given strength will vary as the inverse of the light power. Obviously this time-power relationship will break down at high powers when the buildup time approaches the material characteristic times, i.e., the charge recombination and diffusion times. Various experiments have already been conducted in different insulating crystals $\left(\mathrm{LiNbO}_{3},{ }^{4} \mathrm{BSO}^{5-8} \mathrm{BaTiO}_{3},{ }^{9,10} \mathrm{SBN}^{11}\right)$ with either nanosecond or picosecond pulses.

Our main interest is in sillenite crystals, such as $\mathrm{Bi}_{12}(\mathrm{Si}, \mathrm{Ge}, \mathrm{Ti}) \mathrm{O}_{20}$ because of their high sensitivity. In this paper we investigate the photorefractive effect generated by two interfering 25-psec pulses. The grating time evolution is continuously analyzed from a few picoseconds to a tenth of a millisecond. Section 2 briefly presents the experimental time analysis from a few picoseconds to a few nanoseconds, when the grating evolution is probed by a third 25-psec pulse. In Section 3 we investigate the grating formation from $1 \mathrm{nsec}$ to $0.1 \mathrm{msec}$ by probing with a low-power quasi-continuous argon laser. The photorefractive grating builds up after illumination in two steps. The relative magnitudes of the fast (nanosecond) and slow (microsecond) contributions vary strongly with fluence. This behavior, together with the photocurrent time evolu- tion is explained by means of a band-transport model with shallow traps. Numerical values of mobility, recombination time, and diffusion length of photoexcited charge carriers are derived. We then address, in Section 4, the question of the relationship between parameters determined in either picosecond or quasi-continuous photorefractive experiments. We show that there is total continuity from quasi-continuous to picosecond regimes in sillenite crystals.

\section{PRELIMINARY RESULTS}

We previously predicted and experimentally demonstrated that photorefractive gratings in BSO crystals grow and develop after illumination by a picosecond interference pattern. ${ }^{8}$ Our analysis is based on the consideration of the ratio of the diffusion time $\tau_{d}$ to the recombination time $\tau_{R}$ of the charge carriers. From the band-transport model, one obtains

$$
\tau_{d} / \tau_{R}=(\Lambda / 2 \pi L)^{2},
$$

where $L$ is the diffusion length of the photoexcited charge carriers and $\Lambda$ is the spacing of the grating induced by the interference of two coherent light beams. We previously carried out measurements of $L$ in various sillenite crystals. ${ }^{12}$ The measurements indicated that $\tau_{d}$ can easily be made smaller than $\tau_{R}$ by a correct choice of $\Lambda$. Under these conditions a sinusoidal illumination of the crystal by two interfering pulses when duration $\tau_{L}$ is much shorter than $\tau_{R}$ and $\tau_{D}$ leads to a spatial distribution of charges in both free carriers and deep traps. Because diffusion is much faster than recombination, after illumination an efficient photorefractive effect builds as the modulation of the carrier distribution is washed out, leaving only the ion grating and the subsequent space-charge field. A steadystate regime is reached when the diffusion process is 


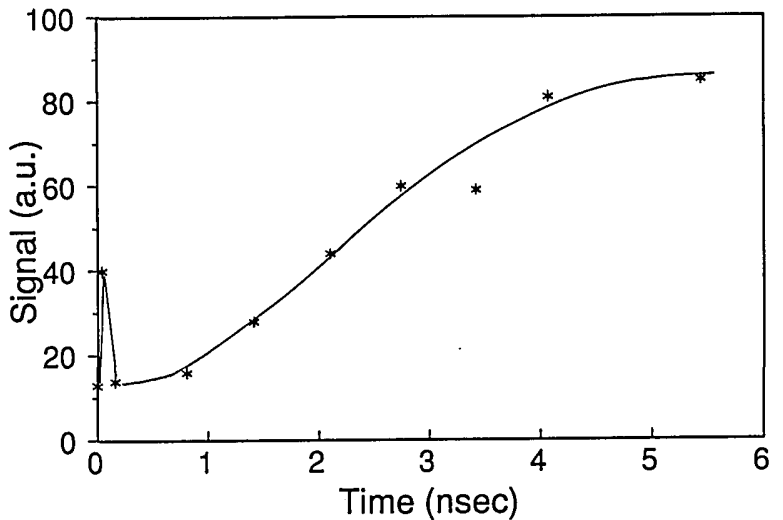

Fig. 1. Phase-conjugate signal versus delay between writing and probe beams.

balanced by the drift of the carriers in the induced spacecharge field.

An experimental demonstration was given by means of a degenerate four-wave mixing scheme with 28-psecduration pulses. The temporal evolution of the grating was analyzed by time delaying the counterpropagating pulse. Figure 1 presents a typical result, showing the phase-conjugate signal, which is proportional to the square of the grating strength, as a function of the delay. The sharp peak at zero delay corresponds to an instantaneous free-carrier nonlinearity. ${ }^{7,8}$ The photorefractive signal starts developing and grows within a few nanoseconds, well after the end of illumination. We verified that it still exists after a few seconds (usual memory effect).

Because the space-charge field is induced by migration of the free carriers, an analog behavior has to be observed on the photocurrent generated by a single, uniform pulse illuminating the crystal, biased by an external electric field. A first analysis ${ }^{8}$ indicates a multiexponential decay time that is attributed to a complex impurity level structure.

\section{PICOSECOND INVESTIGATION OF BSO CRYSTALS}

The aim of this study is to investigate more deeply the time behavior of the photorefractive grating from a few picoseconds to milliseconds and to relate it to the photocurrent evolution.

For all the picosecond measurements reported here, we use an undoped $n$-BSO sample from Crystal Technology. The entrance face is a (110) plane. When a grating is generated by beam interference, its wave vector is along the [110] direction. This orientation is chosen because it minimizes beam-coupling effects in BSO. The crystal thickness along the [110] beam propagation direction, is $e=2 \mathrm{~mm}$, and its entrance surface is $S=1 \mathrm{~cm}^{2}$. The laser source is a passively mode-locked Nd:YAG laser from Brassart-Michelet Industries. Single pulses are extracted by using a Pockels cell and then are amplified. After frequency doubling, the average pulse width is $25 \mathrm{psec}$ and its energy is $1 \mathrm{~mJ}$. The laser can be operated as a single shot or at a repetition rate ranging from 1 to $10 \mathrm{~Hz}$. The crystal absorption at wavelength $\gamma=532 \mathrm{~nm}$ is $\alpha=2.5 \mathrm{~cm}^{-1}$.

\section{A. Photocurrent Analysis}

The photocurrent generated by uniform illumination of the crystal by a single picosecond pulse is studied by applying a constant voltage $V=2 \mathrm{kV}$ to silver-painted electrodes on the $(\overline{1} 10)$ faces of the crystal and by observing the voltage drop on the $50-\Omega$ impedance of a fast oscilloscope with a coupled digitizing camera system $(500 \mathrm{MHz}$, Tektronix Inc.). Figure 2 shows the time evolution of the photocurrent generated by a 25 -psec pulse. One sees a nonexponential decay with two quite different regimes, one in the nanosecond range, the other in the submillisecond range. Multiexponential decay of the photocurrent can be accounted for by considering multiple trapping centers. $^{13,14}$

Measurements of the fast decay time (Fig. 2) give $\tau_{+} \approx 4 \mathrm{nsec}$. In early experiments with light pulses of 10 -nsec duration, ${ }^{6}$ we saw this fast decay process but could not resolve $\tau_{+}$. For a pulse fluence $E=12 \mathrm{~mJ} \mathrm{~cm}^{-2}$, the peak value of the photovoltage is $v=370 \mathrm{mV}$. If we assume a quantum efficiency ${ }^{15,16}$ close to 1 , the density of photoexcited carriers averaged over the crystal thickness is $n_{0}(t=0) \approx 5 \times 10^{14} \mathrm{~cm}^{-3}$. A straightforward calculation gives an estimate of the charge mobility, $\mu \approx$ $0.25 \mathrm{~cm}^{2} \mathrm{~V}^{-1} \mathrm{sec}^{-1}$. Note that for a quantum efficiency of 0.5 we would obtain $n_{0}(0) \approx 2.5 \times 10^{14} \mathrm{~cm}^{-3}$ and $\mu \approx$ $0.5 \mathrm{~cm}^{2} \mathrm{~V}^{-1} \mathrm{sec}^{-1}$. We will discuss the mobility value in Subsection 3.B.1 and Section 4.

We also estimate the ratio $A / B \approx 50$ of the peak photovoltage $A$ (at $t \approx 0$ ) and the voltage $B$ that corresponds to the maximum of the slow component. $B$ is obtained by measuring the amplitude of the photocurrent at $t=$ $50 \mathrm{nsec}$, well before the slow decay.

The photocurrent time evolution reflects the chargecarrier kinetics for a constant electric field only. Experiments conducted with low-power cw lasers showed that this is generally not the case. Indeed, electrode shadowing and nonuniform illumination result in a nonuniform electric-field distribution inside the crystal. Charge redistribution will thus change this field repartition with the dielectric relaxation time constant.

In our picosecond experiments, at the photocurrent peak the dielectric relaxation time is $\tau_{\mathrm{di}}(0)=\left(\varepsilon / n_{0}(0) e \mu\right) \approx$ $0.25 \mu \mathrm{sec}$, which is much larger than $\tau_{+}$(where $\varepsilon$ is the static dielectric constant). Therefore the fast decay reflects the charge density evolution. This is not true at a

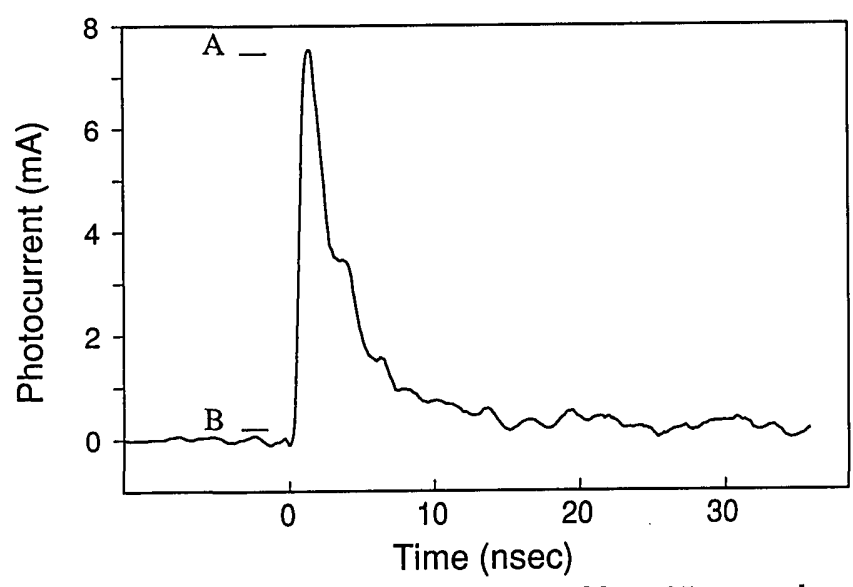

Fig. 2. Kinetics of photocurrent generated by a 25-psec pulse. 


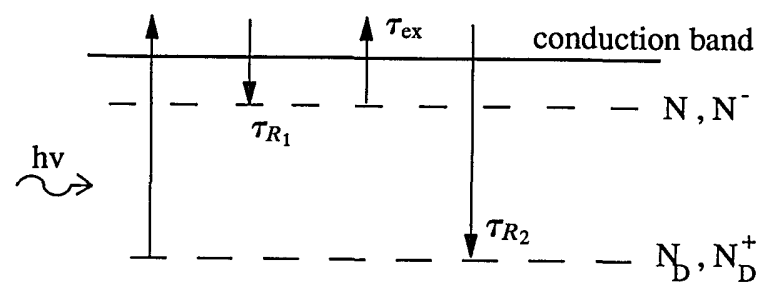

valence band

Fig. 3. Band diagram used for model. $N_{D}$ is the total density of deep centers, $N_{\mathrm{D}}{ }^{+}$the density of ionized deep centers. $N$ is the total density of shallow traps and $N^{-}$the density of shallow traps occupied by an electron. $\tau_{R_{1}}$ and $\tau_{R_{2}}$ are the recombination time constants on shallow and deep traps, respectively; $\tau_{\mathrm{ex}}$ is the thermal excitation time constant.

later time. Indeed, at the beginning of the slow decay when the charge density is reduced by 50 only, $\tau_{\mathrm{di}} \approx$ $12.5 \mu \mathrm{sec}$, which is of the same order or even shorter than the observed photocurrent decay times. To alleviate this problem, we carried out a photocurrent measurement at an extremely low fluence to strongly enlarge the dielectric relaxation time. However, for observation of this weak photocurrent a large-load resistor is required. The reduced frequency bandwith permits measurement of the slow time constant only. One obtains $\tau_{-} \approx 300 \mu \mathrm{sec}$. This is close to the value of $500 \mu \mathrm{sec}$ that we measured in low-power quasi-continuous experiments with flooding beams providing uniform crystal illumination. ${ }^{12}$

As we will see in the following, for our crystal the consideration of a single level of shallow traps and a single level of deep traps (Fig. 3) will give a correct description of the experimental behavior that shows two well-separated regimes, each approximated by a monoexponential decay of time constants $\tau_{+}=4 \mathrm{nsec}$ and $\tau_{-}=500 \mu \mathrm{sec}$.

The mathematical analysis of the time evolution of the carriers generated by a picosecond pulse is given in Appendix $A$. We find that after illumination ends the evolution of the carrier density $n_{0}$ is described by

$$
\frac{\partial^{2} n_{0}}{\partial t^{2}}+\left(\frac{1}{\tau_{R_{2}}}+\frac{1}{\tau_{R_{1}}}+\frac{1}{\tau_{\mathrm{ex}}}\right) \frac{\partial n_{0}}{\partial t}+\frac{n_{0}}{\tau_{R_{2}} \tau_{\mathrm{ex}}}=0 .
$$

In this equation, $\tau_{\mathrm{ex}}$ is a characteristic time describing thermal emission from shallow traps and $\tau_{R_{1}}$ and $\tau_{R_{2}}$ are the recombination times on shallow and deep traps, respectively.

Experimentally, we first observe that the time evolution of the fast component of the photocurrent does not change with incident energy. This indicates a negligible modification of the neutral shallow trap density, allowing us to consider $\tau_{R_{1}}$ to be a constant. Second, we obtain a peak charge density $\left(n_{0} \approx 2.5 \times 10^{14} \mathrm{~cm}^{-3}\right)$ that is smaller than the density of deep traps ionized in the dark $\left(N_{A} \approx\right.$ $10^{16} \mathrm{~cm}^{-3}$, Ref. 12). Parameter $\tau_{R_{2}}$ can also be taken to be constant. Third, we measure $\tau_{+} \ll \tau_{-}$. Fourth, photocurrent experiments conducted on intentionally doped samples revealed that an increase of shallow traps increases $\tau_{-}$and reduces $\tau_{+}{ }^{17}$
As a consequence, these time constants are mainly governed by $\tau_{R_{1}}$, which permits some simplifications (see Appendix A). One obtains

$$
\begin{aligned}
& \tau_{-}=\tau_{R_{2}}\left(\tau_{\mathrm{ex}} / \tau_{R_{1}}\right) \approx 500 \mu \mathrm{sec}, \\
& \tau_{+}=\tau_{R_{1}} \approx 4 \mathrm{nsec} \text {, } \\
& \left.\begin{array}{l}
A=n_{0}(0) \\
B=n_{0}(0)\left(\tau_{R_{1}} / \tau_{\mathrm{ex}}\right)
\end{array}\right\} \text { with } A / B \approx 50 .
\end{aligned}
$$

The time evolution of the charge carriers can thus be written

$$
n_{0}(t)=n_{0}(0)\left\{\exp \left(\frac{-t}{\tau_{R_{1}}}\right)+\frac{\tau_{R_{1}}}{\tau_{\mathrm{ex}}} \exp \left[-\left(\frac{t \tau_{R_{1}}}{\tau_{R_{2}} \tau_{\mathrm{ex}}}\right)\right]\right\},
$$

and one derives

$$
{ }_{787-\mathrm{x}} \quad \tau_{\mathrm{e}} \approx 200 \mathrm{nsec}, \quad \tau_{R_{2}} \approx 10 \mu \mathrm{sec} .
$$

Time $\tau_{R_{2}}$ is the actual recombination time on deep levels in $\mathrm{BSO}$, independent of the presence of shallow traps.

\section{B. Photorefractive Grating Analysis}

\section{Time Evolution}

Because of experimental limitations on the delay line, four-wave mixing with three picosecond pulses does not permit a study of the grating evolution over long durations. In our previous experiments only the first few nanoseconds were analyzed. ${ }^{8}$

To achieve a detailed investigation of the photorefractive grating behavior over a large time interval, we replace the reading pulse beam by a continuous argon-laser beam. This beam is diffracted under Bragg conditions by the grating that is generated by the two interfering picosecond pulses (Fig. 4). The diffracted signal is detected on photomultiplier D1 (rise time $<2$ nsec) and simultaneously recorded on the $500-\mathrm{MHz}$ fast oscilloscope and on a slower one $(20 \mathrm{MHz}$, Gould Inc.).

In our experiments we always analyze the time evolution of a single grating, that is, a grating generated from the interference pattern of two picosecond pulses, even when

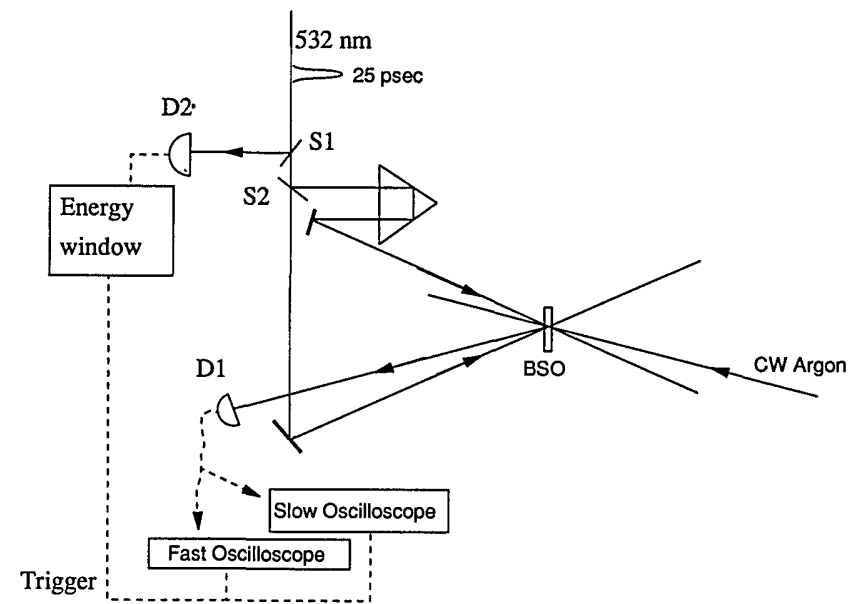

Fig. 4. Experimental setup used for studying kinetics of induced grating. Photomultiplier D1 detects the diffracted signal. The pump fluence is controlled by the beam splitter S1 and photodiode D2. 


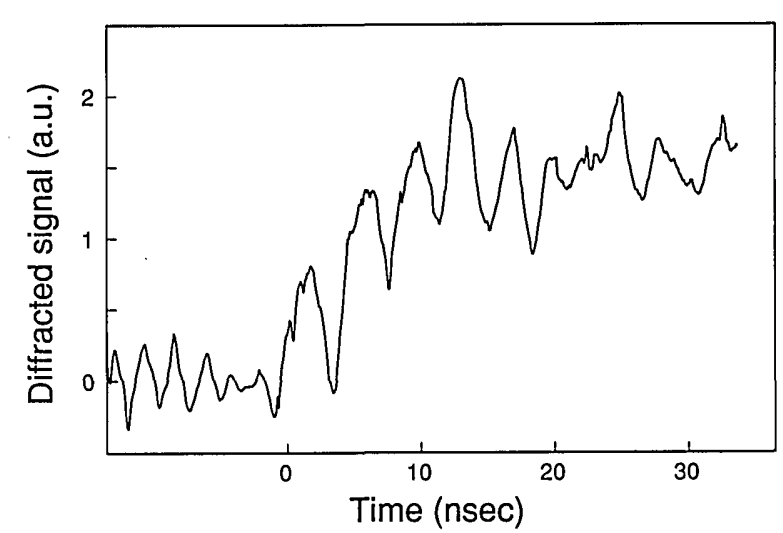

Fig. 5. Kinetics of fast component of the diffracted signal.

the laser is operating at a repetition rate of $10 \mathrm{~Hz}$. For this analysis we set the power of the reading argon laser so that the grating decay time is approximately 1-10 msec.

A reference beam is extracted by beam splitter $\mathrm{S} 1$ and received by photodiode $\mathrm{D} 2$. When the output signal is in a predefined energy window, the data from the photomultiplier are acquired and stored in the two oscilloscopes. Results are averaged on 64 shots that have nearly the same energy. The two interfering pulses are derived by means of beam splitter $\mathrm{S} 2$ and are recombined to produce an interference pattern with modulation index $m=0.5$ and spacing $\Lambda=8 \mu \mathrm{m}$. A delay line on one of the beam paths permits the equalization of the optical paths. This is checked by monitoring the second-harmonic energy produced by an angle-matched KDP crystal.

Figure 5 shows a typical diffracted signal received on the fast oscilloscope. The average fluence in the crystal is $E=2.6 \mathrm{~mJ} \mathrm{~cm}^{-2}$. The signal rise time is $\sim 7 \mathrm{nsec}$, confirming that seen in previous experiments. ${ }^{18}$

Figure 6 shows the evolution of the diffracted signal analyzed on a much longer time scale and its evolution with the pulse fluence. One easily sees a second contribution to the grating buildup, the time constant of which varies from $3 \mu \mathrm{sec}$ to less than $1 \mu \mathrm{sec}$, depending on the energy. The relative magnitudes of the fast and slow contributions to the grating strength vary strongly with energy. At low fluence, $E=0.4 \mathrm{~mJ} \mathrm{~cm}^{-2}$ (Fig. 6a), the fast process makes only a small contribution to the total photorefractive grating strength. As the incident energy increases, this process participates to a greater and greater extent (Fig. 6b), finally to generate the full grating in a few nanoseconds at high fluence $E=9.6 \mathrm{~mJ} \mathrm{~cm} \mathrm{~cm}^{-2}$ (Fig. 6c).

The basic representation of Fig. 3 enables us to obtain physical understanding of these evolutions in time and with energy. At low fluences few charges are photoexcited from deep levels during the 25-psec illumination. They diffuse to an extremely small degree before being trapped in shallow levels $\left(\tau_{R_{1}} \approx 4 \mathrm{nsec}\right)$. One thus observes two charge gratings with slightly different modulations, one in the shallow traps, and the other in the deep traps; the result is a small space-charge field. The charges are then released by thermal excitation $\left(\tau_{\mathrm{ex}} \approx\right.$ 200 nsec). Through successive re-excitations from and trappings in shallow levels they diffuse, reducing the modulation of this charge grating until final recombination in deep traps occurs. The ratio $\tau_{-} / \tau_{\mathrm{ex}} \approx 2 \times 10^{3}$ gives an estimate of the number of thermal excitation-trapping processes in shallow levels. The steady-state photorefractive grating results from the space-charge field created by spatial redistribution of charges in the deep traps as in quasicontinuous excitations with low-power lasers. However, in the case of short-pulse excitation at extremely low energy, the buildup speed corresponds to the time required for reducing to nearly zero the modulation of the chargecarrier grating (coupled with shallow traps), and the grating strength may be smaller than the diffusion field.

Increasing the fluence generates more and more carriers. Even if they still diffuse little before being trapped in shallow levels, the magnitude of the induced spacecharge field increases. The photorefractive grating is then partly or totally built up during the first nanoseconds. When the charges are released from shallow traps by thermal excitation, there is little or no more reduction of this charge-grating modulation as the diffusion process is balanced by drift in the induced field.

The theoretical analysis of the time evolution of the photorefractive grating was carried out by adding a single shallow level to the basic material equations of the usual band transport model. The mathematical development is given in Appendix B. The basic equations (rate, continuity, and current equations and Poisson's law) are linearized by using the assumption that the modulation of the illuminating fringe pattern is small enough to ensure sinusoidal variations of any spatially varying functions in the equations. A numerical integration of the material differential equations is performed with our experimental data deduced from the photocurrent and absorption experi-
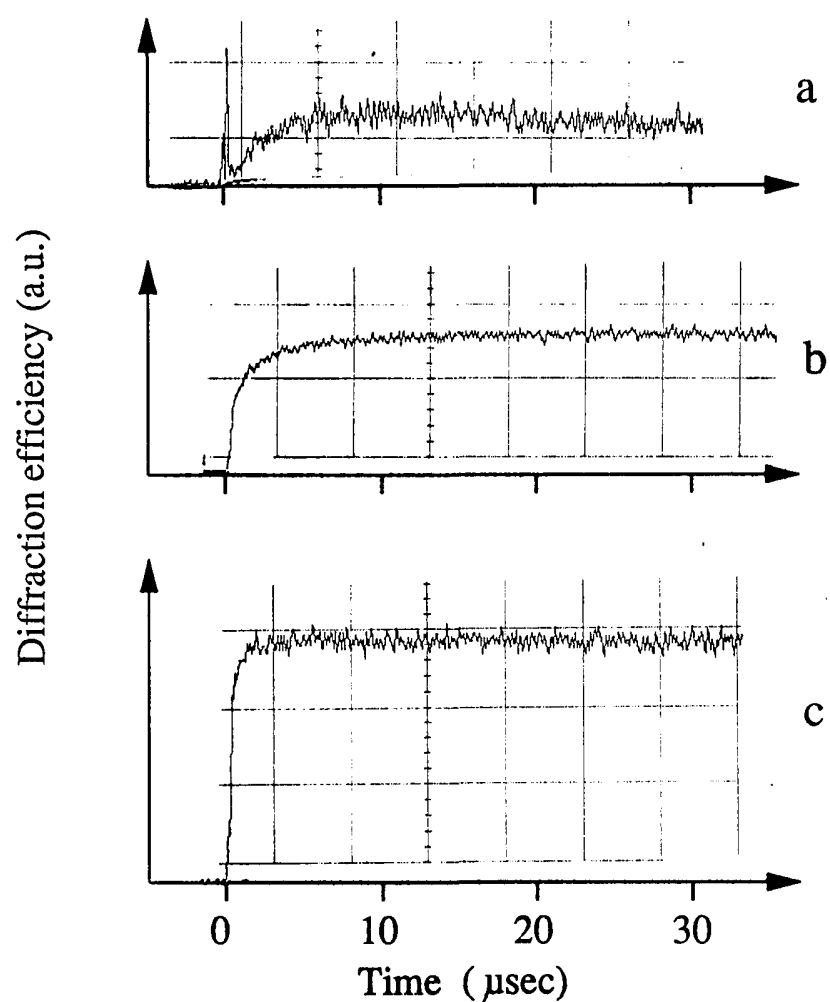

Fig. 6. Irinetics of diffracted signal for three pump fluences: a, $0.4 \mathrm{~mJ} / \mathrm{cm}^{2}$. The sharp peak visible at $t=0$ in a corresponds to light scattered from the writing beams and does not belong to the diffracted signal. b, $2.6 \mathrm{~mJ} / \mathrm{cm}^{2} ; \mathrm{c}, 9.6 \mathrm{~mJ} / \mathrm{cm}^{2}$. 


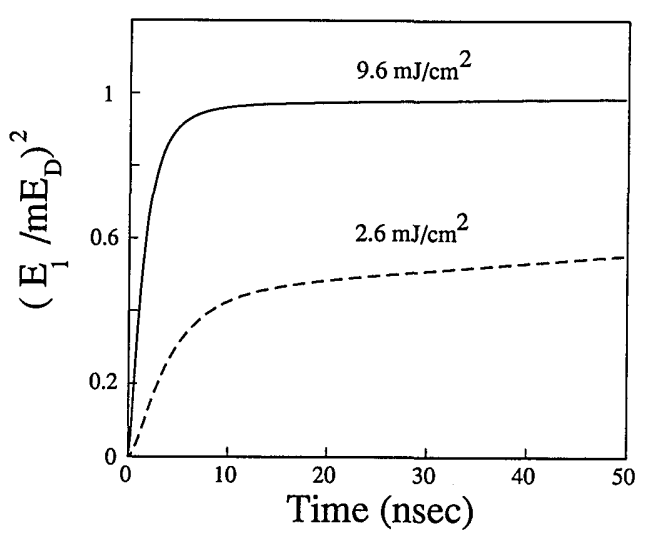

Fig. 7. Computer simulations of the kinetics of the diffracted signal for two pump fluences, $2.6 \mathrm{~mJ} / \mathrm{cm}^{2}$ (dashed curve) and $9.6 \mathrm{~mJ} / \mathrm{cm}^{2}$ (solid curve).

ments. The best agreement with the experiments is obtained for $\mu \approx 0.5 \mathrm{~cm}^{2} \mathrm{~V}^{-1} \mathrm{sec}^{-1}$ and $\tau_{R_{2}} \approx 10 \mu \mathrm{sec}$.

In the calculation the neutral deep-level concentration $\left(N_{D}-N_{D}{ }^{+}\right)$is considered to be much larger than the photoexcited charge density, which is largely verified if one assumes the usual value $\left(N_{D}-N_{D}{ }^{+}\right)=10^{19} \mathrm{~cm}^{-3}$. Note that for high fluences the density of photogenerated charge carriers can approach the concentration of deep traps ionized in the dark. As a consequence the recombination time $\tau_{R_{2}}$ varies with energy and time (see Appendix B).

Figure 7 shows the computed fast kinetics of the photorefractive effect. Its time constant is similar to what we experimentally measure ( $\sim 7 \mathrm{nsec})$. Figure 7 also confirms that at high fluence the maximum space-charge field is reached during the first nanoseconds. At $2.6 \mathrm{~mJ} /$ $\mathrm{cm}^{2}$, Figs. 7 and 8 show that the ratio of the relative contributions to the photorefractive effect is $\sim 0.4$.

At $0.4 \mathrm{~mJ} \mathrm{~cm}^{-2}$ (Fig. 8), the fast contribution represents 2.5\% only. Figure 8 shows that the slow buildup time ( $\leqslant 2 \mu \mathrm{sec}$ ) is comparable with that which we observe experimentally. The maximum space-charge field is equal to $m E_{D}$, the product of the diffusion field times the fringe modulation. The calculated dependence on fluence confirms the analysis presented above when we discussed the experiments.

Theoretical analysis also shows that, when the photorefractive grating is fully built during the first nanoseconds, there is a slow decay of the space-charge field as the shallow traps are emptied and carriers are recombined in deep levels. Indeed (see Appendix B), the recombination of the charge carriers in the bright fringes is more important than in the dark fringes. During recombination the equilibrium between diffusion and drift in the induced space-charge field is broken and is compensated for by a charge flow toward the bright fringes. This process reduces the space-charge field until complete recombination. This behavior exists also in the absence of shallow traps, as previously indicated. ${ }^{18,19}$

In previous studies with either nanosecond ${ }^{6}$ or picosecond $^{8}$ pulses higher values of the mobility were derived ( $\mu>3 \mathrm{~cm}^{2} \mathrm{~V}^{-1} \mathrm{sec}^{-1}, \mu \approx 50 \mathrm{~cm}^{2} \mathrm{~V}^{-1} \mathrm{sec}^{-1}$, respectively). This is because in these analysis it was assumed that the photorefractive grating was generated by total diffusion of the charge carriers in a few nanoseconds. We have shown here that this hypothesis is not required for an explanation of the buildup of the space-charge field to its maximum $m E_{D}$ in few nanoseconds and that the correct value of the mobility is $\sim 0.5 \mathrm{~cm}^{2} \mathrm{~V}^{-1} \mathrm{sec}^{-1}$.

For a recombination time $\tau_{R_{2}} \approx 10 \mu \mathrm{sec}$, the diffusion length for the excitation recombination process in deep traps is

$$
L=\left(k_{B} T \mu \tau_{R_{2}} / e\right)^{1 / 2} \approx 3.6 \mu \mathrm{m} .
$$

This result must be compared with our measurement for this same sample in quasi-continuous experiments with low-power lasers. The analysis of the photorefractive grating decay time under uniform low-power illumination as a function of the magnitude of a static electric field gave us a diffusion length ${ }^{12} L_{\mathrm{CW}}=(3.4 \pm 0.7) \mu \mathrm{m}$ of the same order of magnitude. This will be discussed in Section 4.

\section{Grating Efficiency}

We also measure the efficiency of picosecond pulses in writing the grating. The diffracted signal of the reading continuous argon laser is taken when the steady-state regime is reached. For example, at $E \approx 5 \mathrm{~mJ} \mathrm{~cm}^{-2}$, the measurement is made $50 \mu \mathrm{sec}$ after the writing by the two picosecond pulses, that is, long after the steady state is reached. When the diffraction efficiency is defined as the ratio of the diffracted signal to the one transmitted by the BSO crystal, one obtains $\eta \approx 6 \times 10^{-4}$. A straightforward calculation, with the photoinduced space-charge field assumed to be equal to the diffusion field (fringe spacing $\Lambda=8 \mu \mathrm{m}$, refractive index $n \approx 2.6, r_{41} \approx 4 \times$ $10^{-12} \mathrm{pm} \mathrm{V} \mathrm{V}^{-1}$ as is generally accepted), for a 2 -mm-thick crystal yields $\eta_{\text {th }} \approx 2 \times 10^{-5}$, which is more than one order of magnitude smaller than measured. Obviously the simultaneous recording of photorefractive and absorption gratings could lead to such an increase in diffraction efficiency. However, this seems unlikely because recent time-resolved experiments in a BGO crystal $^{20}$ showed that the induced absorption builds up in less than 1 nsec. No grating is seen on this time scale in our experiments. Nevertheless, to be fully convinced, we studied the kinetics of the induced absorption in our BSO crystal. The transmission of the sample is monitored by using a weak 25-psec pulse. Its fluence $\left(\sim 0.9 \mu \mathrm{J} \mathrm{cm}{ }^{-2}\right)$ is low enough to introduce no change in the absorption. The 25-psec

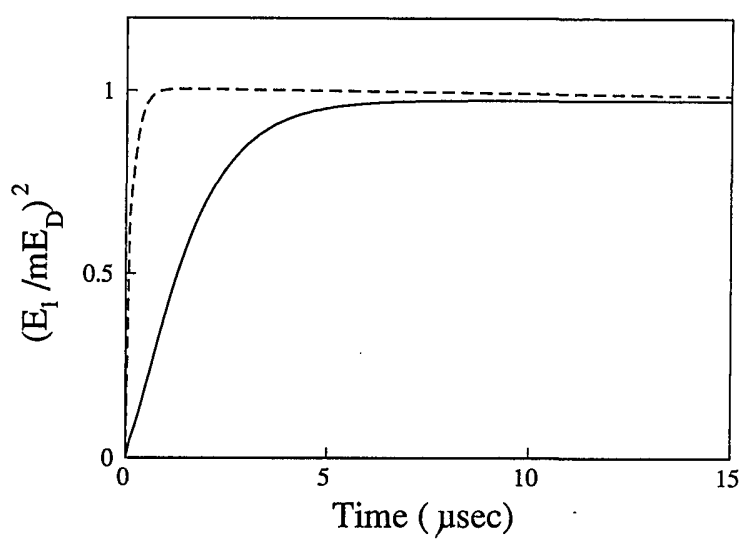

Fig. 8. Computer simulations of the kinetics of the diffracted signal on microsecond scale for two pump fluences, $0.4 \mathrm{~mJ} / \mathrm{cm}^{2}$ (solid curve) and $2.6 \mathrm{~mJ} / \mathrm{cm}^{2}$ (dashed curve). 
pump has a fluence of $9 \mathrm{~mJ} \mathrm{~cm}^{-2}$. An optical delay is introduced on the probe-beam path for the analysis of the time evolution of the crystal transmission. The relative change in transmission $\Delta S / S$ is defined as the difference in the probe transmission without and with the pump divided by the probe transmission without the pump. At zero delay one measures $\Delta S / S \approx 50 \%$. For a delay smaller than $1 \mathrm{nsec}$, a nearly steady-state value of $30 \%$ is reached; it relaxes in about $10 \mathrm{msec}$. Consequently there is no contribution from an induced absorption grating in the diffracted signal analyzed above (Figs. 5 and 6). Indeed, first, we do not observe any signal when the induced absorption of the crystal varies and, second, the buildup of the grating happens when this induced absorption maintains a constant value.

We do not yet explain this unexpected rise in diffraction efficiency. However, some reasons can be considered. It was recently shown, for example, that high-level illumination gives an unexpected increase, more than 200 -fold, in the photoinduced refractive index change in $\mathrm{LiNbO}_{3} .{ }^{21}$ One could also suspect the validity of using the clamped electro-optic coefficient on a nanosecond time scale. Further analysis must be developed for clarification of these phenomena.

\section{COMPARISON WITH MEASUREMENTS IN THE CONTINUOUS REGIME}

We must now raise the following questions: What do we measure in quasi-continuous experiments with low-power lasers? In particular, how can we compare the diffusion length measured by the analysis of the photorefractive decay time under uniform illumination ${ }^{12,15,16,22}$ and the one deduced from picosecond experiments? What is the mobility-lifetime product to be used in moving-grating experiments ${ }^{23}$ ? To what does the mobility measured in ac-field experiments ${ }^{24}$ correspond? In this section we propose some answers to these questions.

The theoretical model of the photorefractive effect generated by low-power cw light beams, with the existence of a shallow level taken into account is presented in Appendix C. This analysis shows that for steady state nothing is changed by introducing this level for the density of free carriers and the photocurrent as well.

For the spatially modulated quantities, the amplitude $n_{1}$ of the free-carrier grating is much smaller than that $\left(N_{1}{ }^{-}\right)$ given by trapping in the shallow level. Indeed $n_{1}=N_{1}$ $\left(\tau_{R_{1}} / \tau_{\text {ex }}\right)$, where $\tau_{\text {ex }}$, the thermal excitation time, is much longer than the recombination time $\tau_{R_{1}}$ in shallow traps. As a consequence the space-charge field results from two gratings of different strengths, corresponding to charge modulations in deep and shallow traps. The set of equations that describe the grating evolutions (charge gratings and space-charge field) is identical to that obtained in models without shallow levels (see Appendix C). It is interesting to note that a shallow level gives only a reduced apparent mobility $\mu^{\prime}=\mu\left(\tau_{R_{1}} / \tau_{\text {ex }}\right)$ and a larger apparent recombination time $\tau_{-}=\tau_{R_{2}}\left(\tau_{\mathrm{ex}} / \tau_{R_{1}}\right)$ that is the slow decay time of the photocurrent (see Subsection 3.A).

This analysis leads to an important result. The diffusion length measured by decay-time analysis of a photorefractive grating under uniform cw illumination ${ }^{12,15,16,22}$ or obtained by separately measuring the apparent mobility
Table 1. Experimental Results ${ }^{a}$

\begin{tabular}{|c|c|c|c|}
\hline Crystal & $\begin{array}{c}\text { Mobility } \\
{\left[10^{-6} \mathrm{~m}^{2} /(\mathrm{V} \text { sec })\right]}\end{array}$ & $\begin{array}{l}\text { Recombination } \\
\text { Time }(\mu \mathrm{sec})\end{array}$ & $\begin{array}{c}\text { Diffusion } \\
\text { Length }(\mu \mathrm{m}) \\
\end{array}$ \\
\hline $\begin{array}{l}\text { BGO } 1 \\
\text { (undoped) }\end{array}$ & 9 & 22 & 2.3 \\
\hline $\begin{array}{l}\text { BGO } 2 \\
\quad(\text { Fe } 17 \text { parts } \\
\left.\text { in } 10^{6}\right)\end{array}$ & 1.8 & 525 & $4.9^{*}$ \\
\hline $\begin{array}{l}\text { BGO } 3 \\
\text { (Fe } 22 \text { parts } \\
\text { in } 10^{6} \text { ) }\end{array}$ & 0.7 & 650 & $3.4^{*}$ \\
\hline $\begin{array}{l}\text { BGO } 4 \\
\quad(\mathrm{Fe} 50 \text { parts } \\
\left.\text { in } 10^{6}\right)\end{array}$ & 0.44 & 1200 & $3.7^{*}$ \\
\hline
\end{tabular}

${ }^{a}$ An asterisk indicates the parameter that is deduced from the other two.

and recombination time in two-wave mixing with an ac field ${ }^{24}$ is the same as that measured in picosecond experiments. Indeed,

$$
L=\left(k_{\mathrm{B}} T \mu \tau_{R_{2}} / e\right)^{1 / 2}=\left(k_{\mathrm{B}} T \mu^{\prime} \tau_{-} / e\right)^{1 / 2} .
$$

This analysis also shows that the same order of magnitude must be found, whatever the shallow-level concentration. Changing this concentration will modify only $\mu^{\prime}$ and $\tau_{-}$but not their product. This finding is illustrated by the results in Table 1, where the apparent mobilities and recombination times have been determined for four different BGO crystals grown at the Université de Bordeaux, France. BGO 1 is nominally undoped while BGO 2 to BGO 4 are iron doped. The concentrations in the melt are 17 parts in $10^{6}$ for BGO 2,22 parts in $10^{6}(+20$ parts in $10^{6}$ vanadium) for BGO 3 , and 50 parts in $10^{6}$ for BGO 4.

The apparent recombination time constants $\tau_{-}$, deduced from photocurrent experiments, are listed in column 3 of Table 1 . BGO 1 has been studied by the grating-decay-time analysis, ${ }^{12}$ which gives the diffusion length (column 4). A straightforward calculation leads to an estimate of the apparent mobility (column 2).

For BGO 2 to BGO 4, the apparent mobility was measured by studying the time oscillations of the photorefractive gain in two-wave mixing experiments. Indeed, when an ac electric field is applied parallel to the grating wave vector, the sudden change of sign at each half-period produces a drift of the charge grating. This drift creates a running charge grating, moving in the presence of the fixed ionic grating, that makes the space-charge field oscillate. This is easily visible in the intensity of the beam amplified by two-beam coupling experiments. A measurement of the oscillation frequency gives the apparent mobility. By looking at column 2 and 3, one first sees that the more heavily doped the sample, the longer the apparent recombination time constant and the smaller the apparent mobility. Inserting the mobility and time constant values into the diffusion length expression gives the values in column 4 .

It is striking to see that the diffusion length varies quite little for samples with extremely different mobilities and recombination times. This result fully supports our analysis. 


\section{CONCLUSION}

We described an extensive investigation of the formation of photorefractive gratings generated in BSO by picosecond pulses. The buildup starts after illumination. This is possible because the diffusion time is shorter than the recombination time in deep traps. At fluences larger than $5 \mathrm{~mJ} \mathrm{~cm}^{-2}$ the maximum steady-state efficiency is reached in less than 10 nsec. At lower fluences the grating first evolves rapidly ( $<10 \mathrm{nsec}$ ) and then slowly (microseconds) goes to its maximum. The relative contributions of the fast and slow components to the grating strength are determined by the fluence. This behavior as well as the photocurrent time evolution are explained by transient trapping in shallow levels. The modeling by means of a modified band-transport model gives results that corresponds well with the experimental measurements. Moreover, it shows how picosecond experiments in sillenite crystals permit the determination of the actual mobilities, lifetimes, and diffusion lengths of the photoexcited carriers. This determination is feasible because the pulse duration is smaller than both the recombination and the diffusion times. The analysis of the quasicontinuous regime with shallow traps shows what one measures in cw experiments. First one determines the same diffusion length. Then one measures apparent mobilities and recombination times, the values of which depend on shallow-level concentrations. Shallow levels reduce the apparent mobility $\mu^{\prime}$ and increase the apparent recombination time $\tau_{-}$. However, the $\mu^{\prime} \tau_{-}$product remains constant and equal to the product of actual mobility and recombination time for deep traps that are measured in picosecond studies. This explain the strong similarities from sample to sample in quasi-continuous experiments for which the important parameter is the diffusion length. This will not be the case in picosecond experiments for which crystals with few shallow traps permit a fast grating buildup even at low energy or for crystals with high concentrations of shallow traps that exhibit a slow rise of the photo-refractive effect.

In this study we limited ourselves to a single shallowtrap level. Extension to multilevel shallow traps will make the analysis more complicated but will not change the basic idea.

\section{APPENDIX A}

Consider the basic representation of Fig. 4 (above) with one deep center of total concentration $N_{D}$, partly ionized $\left(N_{D}^{+}\right)$, and one shallow trap of total density $N$ that becomes ionized $\left(N^{-}\right)$when trapping electrons. Photoexcitation by a uniform 25-psec pulse at $532 \mathrm{~nm}$ generates electrons that can migrate in the conduction band. Constants $\tau_{R_{1}}$ and $\tau_{R_{2}}$ are the recombination times in shallow and deep traps, respectively, and $\tau_{\mathrm{ex}}$ is a characteristic time that represents thermal emission from shallow traps to the conduction band. With application of a constant electric field $E_{0}$, the evolution of the electron density $n_{0}$ in the conduction band is described by the following equations. For the point just after illumination ends, one obtains

$$
\begin{aligned}
& \frac{\partial N_{0}^{-}}{\partial t}=-\frac{N_{0}^{-}}{\tau_{\mathrm{ex}}}+\gamma_{1} n_{0}\left(N-N_{0}^{-}\right), \\
& \frac{\partial N_{D 0^{+}}}{\partial t}=-\gamma_{2} n_{0} N_{D 0^{+}}, \\
& {N_{D 0^{+}}}^{+}-n_{0}-N_{0^{-}}^{-}-N_{A}=0,
\end{aligned}
$$

where $j_{0}$ is the current density and $N_{A}$ is the density of compensating shallow acceptors that provide charge neutrality. One observes experimentally that the time evolution of the photocurrent does not change with incident energy; thus $N_{0}^{-} \ll N$, and

$$
\tau_{R_{1}}=\frac{1}{\gamma_{1}\left(N-N_{0}^{-}\right)} \approx \frac{1}{\gamma_{1} N} .
$$

In this photocurrent experiment the peak charge density, $n_{0} \approx 5 \times 10^{14} \mathrm{~cm}^{-3}$, is much smaller than $N_{\mathrm{A}} \approx$ $10^{16} \mathrm{~cm}^{-3}$. Thus the concentration $N_{D 0}{ }^{+}$does not really change, and

$$
\frac{1}{\gamma_{2} N_{D 0}{ }^{+}} \approx \frac{1}{\gamma_{2} N_{A}}=\tau_{R_{2}}
$$

is constant.

Under these conditions the system of equations (A.1) leads to an equation that describes the carrier-density evolution, i.e., the photocurrent:

$$
\frac{\partial^{2} n_{0}}{\partial t^{2}}+\left(\frac{1}{\tau_{R_{2}}}+\frac{1}{\tau_{R_{1}}}+\frac{1}{\tau_{\mathrm{ex}}}\right) \frac{\partial n_{0}}{\partial t}+\frac{n_{0}}{\tau_{R_{2}} \tau_{\mathrm{ex}}}=0 .
$$

The general solution is

$$
n_{0}(t)=A \exp \left(-t / t_{+}\right)+B \exp \left(-t / \tau_{-}\right)
$$

with

$$
\begin{aligned}
\frac{1}{\tau_{+}}= & \frac{1}{2}\left\{\left(\frac{1}{\tau_{R_{2}}}+\frac{1}{\tau_{R_{1}}}+\frac{1}{\tau_{\mathrm{ex}}}\right)\right. \\
& \left. \pm\left[\left(\frac{1}{\tau_{R_{2}}}+\frac{1}{\tau_{R_{1}}}+\frac{1}{\tau_{\mathrm{ex}}}\right)^{2}-\frac{4}{\tau_{R_{2}} \tau_{\mathrm{ex}}}\right]^{1 / 2}\right\} .
\end{aligned}
$$

The initial conditions, with $N_{0}{ }^{-}(0)=0$, give $A$ and $B$ :

$$
\begin{aligned}
n_{0}(0) & =A+B, \\
\frac{\partial n_{0}}{\partial t} & =-n_{0}(0)\left(\frac{1}{\tau_{R_{2}}}+\frac{1}{\tau_{R_{1}}}\right)=-\left(\frac{A}{\tau_{+}}+\frac{B}{\tau_{-}}\right) .
\end{aligned}
$$

In our experiments, we measure $\tau_{+} \ll \tau_{-}$; thus Eq. (A.2) leads to

$$
\begin{aligned}
\frac{1}{\tau_{-}} & =\frac{1}{\tau_{\mathrm{ex}}+\left(\tau_{R_{2}} \tau_{\mathrm{ex}} / \tau_{R_{1}}\right)+\tau_{R_{2}}}, \\
\frac{1}{\tau_{+}} & =\frac{1}{\tau_{R_{2}}}+\frac{1}{\tau_{R_{1}}}+\frac{1}{\tau_{\mathrm{ex}}} .
\end{aligned}
$$

Other photocurrent experiments conducted on intentionally doped samples revealed that an increase of shallow traps increases $\tau_{-}$and reduces $\tau_{+}$. Consequently, these time constants are governed mainly by $\tau_{R_{1}}$, giving

$$
\tau_{-}=\tau_{R_{2}} \tau_{\text {ex }} / \tau_{R_{1}}, \quad \tau_{+}=\tau_{R_{1}} .
$$

$$
j_{0}=n_{0} e \mu E_{0}
$$


A simple expression for $A$ and $B$ is now obtained:

$$
A=n_{0}(0), \quad B=n_{0}(0)\left(\tau_{R_{1}} / \tau_{\mathrm{ex}}\right) .
$$

Previously described measurements give $\tau_{+}=\tau_{R_{1}} \approx$ $4 \mathrm{nsec}$ and $A / B \approx 50$, leading to $\tau_{\mathrm{ex}} \approx 200 \mathrm{nsec}$ and $\tau_{-} \approx 500 \mu \mathrm{sec}$, in turn leading to a recombination time constant for deep traps of $\tau_{R_{2}} \approx 10 \mu \mathrm{sec}$.

\section{APPENDIX B}

From the basic representation of Fig. 4, the photorefractive effect generated by short pulses is described by writing the basic material equations (rate, continuity, and current equations and Poisson's law):

$$
\begin{aligned}
\frac{\partial N^{-}}{\partial t} & =\frac{-N^{-}}{\tau_{\mathrm{ex}}}+\gamma_{1} n\left(N-N^{-}\right), \\
\frac{\mathrm{d} N_{D}^{+}}{\partial t} & =-\gamma_{2} n N_{D}^{+}, \\
\operatorname{div} j & =-e\left(\frac{\partial N_{D}^{+}}{\partial t}-\frac{\partial n}{\partial t}-\frac{\partial N^{-}}{\partial t}\right), \\
j & =\mu n e E+\mu k_{B} T \operatorname{grad} n, \\
\operatorname{div} E & =(e / \varepsilon)\left(N_{D}^{+}-n-N^{-}-N_{A}\right) .
\end{aligned}
$$

They are linearized by using the assumption that the modulation is small enough to ensure sinusoidal variations of any spatially varying quantities. We use complex notation and develop all the variables in the form

$$
v(t)=v_{0}(t)+v_{1}(t) \exp (i \mathbf{k} \cdot x)
$$

with $x$ the space coordinate along the $\mathbf{k}$ vector.

For the zeroth order one has the set of equations (A.1) (Appendix A) for no applied field. Here we do not assume a small generation of carriers; thus $\gamma_{2} N_{D 0}{ }^{+}=N_{D 0}{ }^{+} /$ $N_{A} T_{R_{2}}$, where $\tau_{R_{2}}=1 /\left(\gamma_{2} N_{A}\right)$ is the recombination time determined at low fluence in the photocurrent experiments. System (A.1) reduces to

$$
\begin{aligned}
\frac{\partial n_{0}}{\partial t} & =-n_{0}\left(\frac{1}{\tau_{R_{1}}}+\frac{1}{\tau_{R_{2}}} \frac{N_{D 0}{ }^{+}}{N_{A}}\right)+\left(N_{D 0}^{+}-n_{0}-N_{A}\right) \frac{1}{\tau_{\mathrm{ex}}} \\
\frac{\partial N_{D 0}{ }^{+}}{\partial t} & =-\frac{n_{0}}{\tau_{R_{2}}} \frac{N_{D 0^{+}}}{N_{A}}
\end{aligned}
$$

For the first order just after the pulse, one has

$$
\begin{aligned}
j_{1} & =e \mu n_{0} E_{1}+i e \mu n_{1} E_{D}=i e \mu\left(n_{0} E_{1}^{i m}+n_{1} E_{D}\right), \\
\frac{\partial N_{1}^{-}}{\partial t} & =-\frac{N_{1}^{-}}{\tau_{\mathrm{ex}}}+\gamma_{1} n_{1} N-\gamma_{1} n_{0} N_{1}^{-}, \\
\frac{\partial N_{D 1}^{+}}{\partial t} & =-\gamma_{2}\left(n_{1} N_{D 0^{+}}+n_{0}{N_{D 1}}^{+}\right) \\
i k E_{1} & =(e / \varepsilon)\left({N_{D 1}}^{+}-n_{1}-N_{1}^{-}\right) \\
i k j_{1} & =-e\left(\frac{\partial N_{D 1}^{+}}{\partial t}-\frac{\partial n_{1}}{\partial t}-\frac{\partial N_{1}^{-}}{\partial t}\right),
\end{aligned}
$$

where $E_{D}=k\left(k_{B} T / e\right)$ is the diffusion field that corresponds to a charge grating of spacing $\Lambda=2 \pi / k$.
We will now assume that $n_{0} N_{1}^{-} \ll n_{1} N$. This is obviously true at $t=0$, as $N_{1}^{-}=0$.

This system can be rewritten as

$$
\begin{aligned}
\frac{\partial n_{1}}{\partial t}= & -\mu k\left(n_{0} E_{1}^{i m}+n_{1} E_{D}\right)-n_{1}\left(\frac{1}{\tau_{R_{1}}}+\frac{N_{D 0}{ }^{+}}{N_{A}} \frac{1}{\tau_{R_{2}}}\right) \\
& +\frac{N_{1}^{-}}{\tau_{\mathrm{ex}}}-\frac{n_{0} N_{D 1}^{+}}{\tau_{R_{2}} N_{A}}, \\
\frac{\partial{N_{1}^{-}}^{-}}{\partial t}= & \frac{n_{1}}{\tau_{R_{1}}}-\frac{N_{1}^{-}}{\tau_{\mathrm{ex}}}, \\
\frac{\partial N_{D 1}^{+}}{\partial t}= & -\frac{n_{1}}{\tau_{R_{2}}} \frac{N_{D 0}{ }^{+}}{N_{A}}-\frac{n_{0}}{\tau_{R_{2}}} \frac{N_{D 1}^{+}}{N_{A}} .
\end{aligned}
$$

Equations (B.1) and (B.2) are then solved numerically, with the following parameters given by experiment. From photocurrent measurements,

$$
\tau_{R_{1}}=4 \mathrm{nsec}, \quad \tau_{\mathrm{ex}}=200 \mathrm{nsec}, \quad \tau_{R_{2}}=10 \mu \mathrm{sec}
$$

and

$$
\mu=0.5 \mathrm{~cm}^{2} \mathrm{~V}^{-1} \mathrm{sec}^{-1}
$$

From the absorption measurement, $\alpha=2.5 \mathrm{~cm}^{-1}$.

From the parameter measurements in the quasi-continuous regime for this same crystal, ${ }^{12} N_{A}=0.8 \times 10^{16} \mathrm{~cm}^{-3}$.

\section{APPENDIX C}

To describe the photorefractive effect induced by lowpower lasers, we start again with the basic equations (B.1), accounting for the photogeneration of free carriers in the second-rate equation:

$$
\frac{\partial N_{D}^{+}}{\partial t}=-\gamma_{2} n N_{D}^{+}+s I\left(N_{D}-N_{D}^{+}\right),
$$

with $I$ the optical irradiance and $s$ the photoionization cross section of the deep centers.

We introduce the usual simplifications of the quasicontinuous model. Considering that the density of ionized deep traps is not modified by the low illumination and that the quantities that are not spatially modulated (zeroth order) reach their steady state before the photorefractive grating starts developing, we obtain, for the zeroth order,

$$
\begin{aligned}
j_{0} & =\mu n_{0} e E_{0}, \\
N_{0}^{-} & =n_{0}\left(\tau_{\mathrm{ex}} / \tau_{R_{1}}\right), \\
n_{0} & =\tau_{R_{2}} s I\left(N_{D}-N_{A}\right), \\
N_{D 0^{+}} & =n_{0}+N_{0}^{-}+N_{A} .
\end{aligned}
$$

One immediately sees that introducing the shallow level does not change $n_{0}$ and $j_{0}$.

For the first-order quantities, with a continuous illumination pattern of intensity $I_{0}$ and modulation $m$, one obtains 


$$
\begin{aligned}
\frac{\partial N_{1}^{-}}{\partial t} & =-\frac{N_{1}^{-}}{\tau_{\mathrm{ex}}}\left(1+\frac{N_{0}^{-}}{N}\right)+\frac{n_{1}}{\tau_{R_{1}}} \approx-\frac{N_{1}^{-}}{\tau_{\mathrm{ex}}}+\frac{n_{1}}{\tau_{R_{1}}}, \\
\frac{\partial N_{D 1}^{+}}{\partial t} & =\frac{-n_{1}}{\tau_{R_{2}}}-\gamma_{2} n_{0} N_{D 1}^{+}+m \frac{n_{0}}{\tau_{R_{2}}}, \\
i k E_{1} & =(e / \varepsilon)\left(N_{D 1}^{+}-n_{1}-N_{1}^{-}\right), \\
j_{1} & =\mu e\left[n_{1}\left(E_{0}+i E_{D}\right)+n_{0} E_{1}\right] .
\end{aligned}
$$

The photorefractive effect generated by low-power, quasi-continuous lasers has a time evolution slower than $\tau_{\mathrm{ex}} \approx 200$ nsec. Thus

$$
\frac{\partial N_{1}^{-}}{\partial t} \ll \frac{N_{1}^{-}}{\tau_{\mathrm{ex}}} .
$$

One finally obtains a direct relation between the amplitudes of the gratings in free carriers and shallow traps:

$$
n_{1}=N_{1}-\frac{\tau_{R_{1}}}{\tau_{\mathrm{ex}}}
$$

This relation is identical to that obtained for the zeroth order. In the quasi-continuous regime the concentrations of free charges and the populations in the shallow traps are always in equilibrium. Since $\tau_{R_{1}} / \tau_{\text {ex }} \ll 1$ (measured here as $\left.2 \times 10^{-2}\right), n_{1} \ll N_{1}^{-}$and $n_{0} \ll N_{0}^{-}$.

The above equations now become

$$
\begin{aligned}
\frac{\partial N_{D 1}^{+}}{\partial t} & =-\frac{\tau_{R_{1}}}{\tau_{R_{2}} \tau_{\mathrm{ex}}}\left(N_{1}^{-}+\frac{N_{0}^{-} N_{D 1}^{+}}{N_{A}}-m N_{0}^{-}\right), \\
i k E_{1} & =(e / \varepsilon)\left(N_{D 1}^{+}-N_{1}^{-}\right), \\
\frac{\partial N_{D 1}^{+}}{\partial t}-\frac{\partial N_{1}^{-}}{\partial t} & =-i k \mu \frac{\tau_{R_{1}}}{\tau_{\mathrm{ex}}}\left[N_{1}^{-}\left(E_{0}+i E_{D}\right)+N_{0}^{-} E_{1}\right] .
\end{aligned}
$$

The system of equations (C.1) is the usual one for a single deep level and one kind of charge carrier. The apparent mobility $\mu^{\prime}=\mu\left(\tau_{R_{1}} / \tau_{\mathrm{ex}}\right)$ is weaker, corresponding to electrons moving in the shallow traps, while the apparent recombination time $\tau_{-}=\tau_{R_{2}}\left(\tau_{\mathrm{ex}} / \tau_{R_{1}}\right)$ is larger. Note that it corresponds to the time constant of the slow component of the photocurrent generated with short pulses.

As $\mu^{\prime} \tau_{-}=\mu \tau_{R_{2}}$, one of course expects the same value for the diffusion length, which is independent of shallow levels. Increasing the density of shallow traps will reduce $\mu^{\prime}$ and increase $\tau_{-}$without changing the product.

\section{ACKNOWLEDGMENTS}

We thank the Direction des Recherches, Etudes et Techniques for its interest and for partly supporting this research. We are also indebted to E. Brotons for his participation in the initial photorefractive measurements and to $\mathrm{Ph}$. Delaye for the nonlinear absorption measurements.

\section{REFERENCES}

1. J. P. Huignard and G. Roosen, "Photorefractive materials for optical processing," in Nonlinear Optics: Materials and Devices, Vol. 7 of Proceedings in Physics, C. Flytzanis and J. L. Oudar, eds. (Springer, New York, 1985), pp. 128-141.

2. P. Günter and J. P. Huignard, eds., Photorefractive Materials and Their Applications I and II, Vols. 61 and 62 of Topics in Applied Physics (Springer-Verlag, Berlin, 1988, 1989).
3. Digest of Topical Meeting on Photorefractive Materials, Effects, and Devices II (Optical Society of America, Washington, D.C., 1990).

4. C. T. Chen, D. M. Kim, and D. von der Linde, "Efficient pulsed photorefractive process in $\mathrm{LiNbO}_{3}: \mathrm{Fe}$ for optical storage and deflection," IEEE J. Quantum Electron. QE-16, 126 (1980).

5. J. P. Huignard and J. P. Herriau, "Nanosecond four-wave mixing and holography in BSO crystals," Appl. Opt. 20, 2173 (1981).

6. G. Le Saux, G. Roosen, and A. Brun, "Observation and analysis of the fast photorefractive process in BSO," Opt. Commun. 56, 374 (1986).

7. J. L. Ferrier, J. Gazengel, X. Nguyen Phu, and G. Rivoire, "Picosecond holography and four-wave mixing in BSO," Opt. Commun. 58, 343 (1986).

8. J. M. C. Jonathan, G. Roosen, and Ph. Roussignol, "Timeresolved buildup of a photorefractive grating induced in $\mathrm{Bi}_{12} \mathrm{SiO}_{20}$ by picosecond light pulses," Opt. Lett. 13, 224 (1988).

9. L. K. Lam, T. Y. Chang, J. Feinberg, and R. W. Hellwarth, "Photorefractive index gratings formed by nanosecond optical pulses in $\mathrm{BaTiO}_{3}$," Opt. Lett. 6, 475 (1981).

10. A. L. Smirl, K. Bohnert, G. C. Valley, R. A. Mullen, and Th. F. Boggess, "Formation, decay, and erasure of photorefractive gratings written in barium titanate by picosecond pulses," J. Opt. Soc. Am. B 6, 606 (1989).

11. B. Monson, G. J. Salamo, A. G. Mott, M. J. Miller, E. J. Sharp, W. W. Clark III, G. L. Wood, and R. R. Neurgaonkar, "Selfpumped phase conjugation with nanosecond pulses in strontium barium niobate," Opt. Lett. 15, 12 (1990).

12. G. Pauliat, J. M. C. Jonathan, M. Allain, J. C. Launay, and G. Roosen, "Determination of the photorefractive parameters of $\mathrm{Bi}_{12} \mathrm{GeO}_{20}$ crystals using transient grating analysis," Opt. Commun. 59, 266 (1986).

13. G. Le Saux and A. Brun, "Photorefractive material response to short pulse illuminations," IEEE J. Quantum Electron. QE-23, 1680 (1987).

14. J. L. Ferrier, J. Gazengel, and G. Rivoire, "Ultrafast photorefractive process in BSO," Digest of Topical Meeting on Photorefractive Materials, Effects, and Devices (Optical Society of America, Washington, D.C., 1987), p. 93.

15. P. A. M. dos Santos, P. M. Gargia, and J. Frejlich, "Transport length, quantum efficiency, and trap density measurement in $\mathrm{Bi}_{12} \mathrm{SiO}_{20}$," J. Appl. Phys. 66, 247 (1989).

16. F. P. Strohkendl, P. Tayebati, and R. W. Hellwarth, "Comparative study of photorefractive $\mathrm{Bi}_{12} \mathrm{SiO}_{20}$ crystals," J. Appl. Phys. 66, 6024 (1989).

17. G. Le Saux, J. C. Launay, and A. Brun, "Transient photocurrent induced by nanosecond light pulses in BSO and BGO," Opt. Commun. 57, 166 (1986).

18. J. M. C. Jonathan, Ph. Roussignol, and G. Roosen, "Photorefractive grating build-up by a 28 ps light pulse in BSO," J. Phys. C2, Suppl. 6, 49, C2-267, (1988).

19. L. B. Au, "Response of photorefractive materials for given initial conditions: an analytical solution," Opt. Commun. 72, 265 (1989).

20. M. Sylla, D. Rouede, R. Chevallier, N. P. Xuan, and G. Rivoire, "Nonlinear absorption in BSO, BGO crystals induced by picosecond pulses," in Digest of Topical Meeting on Photorefractive Materials, Effects, and Devices II (Optical Society of America, Washington, D.C., 1990), p. 193.

21. O. Althoff and E. Krätzig, "Strong light induced refractive index changes in $\mathrm{LiNbO}_{3}$," in Nonlinear Optical Materials III, P. Günter, ed., Proc. Soc. Photo-Opt. Instrum. Eng. 1273 (to be published).

22. R. A. Mullen and R. W. Hellwarth, "Optical measurement of the photorefractive parameters of $\mathrm{Bi}_{12} \mathrm{SiO}_{20}$," Appl. Phys. 58, 40 (1985).

23. Ph. Refregier, L. Solymar, H. Rajbenbach, and J. P. Huignard, "Two-beam coupling in photorefractive $\mathrm{Bi}_{12} \mathrm{SiO}_{20}$ crystals with moving grating: theory and experiments," J. Appl. Phys. 58, 45 (1985).

24. G. Pauliat, A. Villing, J. C. Launay, and G. Roosen, "Optical measurements of charge-carrier mobilities in photorefractive sillenite crystals," J. Opt. Soc. Am. B 7, 1481 (1990). 